Provided for non-commercial research and education use. Not for reproduction, distribution or commercial use.

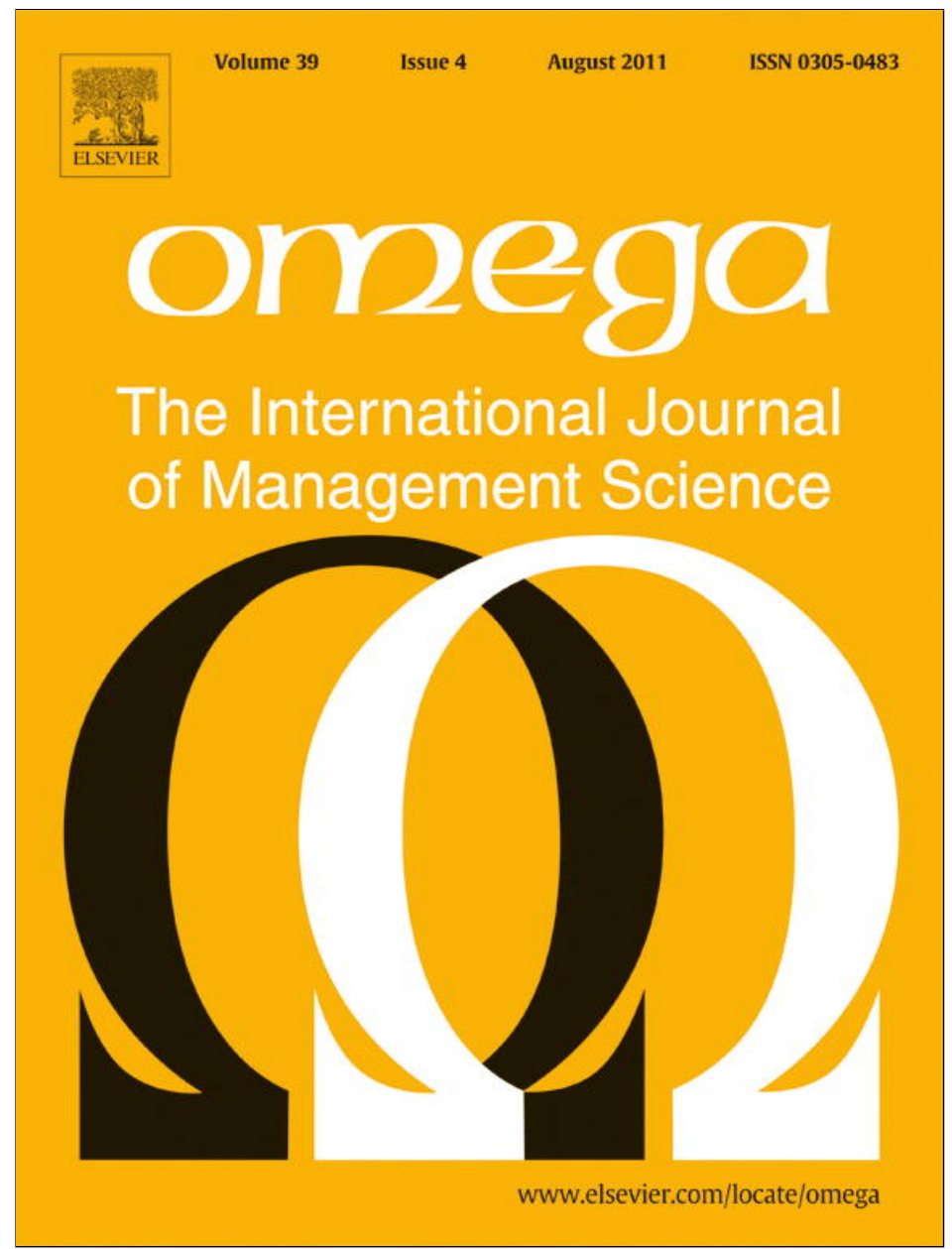

This article appeared in a journal published by Elsevier. The attached copy is furnished to the author for internal non-commercial research and education use, including for instruction at the authors institution and sharing with colleagues.

Other uses, including reproduction and distribution, or selling or licensing copies, or posting to personal, institutional or third party websites are prohibited.

In most cases authors are permitted to post their version of the article (e.g. in Word or Tex form) to their personal website or institutional repository. Authors requiring further information regarding Elsevier's archiving and manuscript policies are encouraged to visit:

http://www.elsevier.com/copyright 


\title{
Evaluation of performance of European cities with the aim to promote quality of life improvements
}

\author{
Paulo Morais*, Ana S. Camanho \\ Faculdade de Engenharia da Universidade do Porto, Departamento de Engenharia Industrial e Gestão (DEIG), Rua Roberto Frias, 4200-465 Porto, Portugal
}

\section{A R T I C L E I N F O}

\section{Article history:}

Received 12 February 2010

Accepted 10 September 2010

Processed by B. Lev

Available online 17 September 2010

\section{Keywords:}

DEA

Quality of life

Urban Audit

Virtual weight restrictions

Composite indicators

\begin{abstract}
A B S T R A C T
This paper explores the possibilities presented by DEA to assess quality of life and evaluate the performance of city managers in what concerns the promotion of urban quality of life. Using the data provided by the Urban Audit program, from the European Union, we defined the city profile regarding quality of life for 206 cities. Two approaches are presented: the construction of a composite indicator of quality of life and the assessment of local management performance, contextualised by the GDP per capita to measure the ability of local authorities to promote quality of life given the economic condition of the country. The results identify the cities with urban best practices and present a model of intervention for the cities considered inefficient, based on benchmarking principles.
\end{abstract}

(c) 2010 Elsevier Ltd. All rights reserved.

\section{Introduction}

The evaluation of the urban quality of life (QoL) is an issue with growing importance in the scientific literature. Several authors, from different academic backgrounds, have approached this theme. Contributions from diverse disciplines illustrate the complexity of this phenomenon: sociology, geography, economy, public health, transport or environment engineering are only some of the possible references that show the interest this issue raises in different areas of knowledge. The multidisciplinary views show, on one hand, the scientific wealth of this issue, but it raises, on the other hand, research constrains as it is difficult to reach a consensual concept of quality of life, as well as the underlying dimensions that should be used for its assessment. In the scientific literature that addresses this theme, the lack of agreement is well shown.

The concern of administrative authorities raised by the growth of people living in urban contexts has been followed by the scientific literature. One of the reasons is that "politicians, policymakers and planners are constantly faced with decisions on environmental, social and economic issues, directly linked with quality of life, at a national, regional, urban and neighbourhood level" [21, p. 2]. This is why quality of life is a reality that has also been demanding increased attention from the authorities of European institutions. A recent resolution of the European Parliament from 2005, considering the urban dimension within

\footnotetext{
* Corresponding author: Tel.: +351913477 159.

E-mail addresses: pauloteixeirademorais@gmail.com (P. Morais), acamanho@fe.up.pt (A.S. Camanho).
}

the process of the enlargement, explains that "towns and cities and urban agglomerations or areas, where $78 \%$ of the European Union population is concentrated, are the place where both the most complex and the most common problems are concentrated (social exclusion, spatial and ethnic segregation, housing shortages, insecurity, drugs, pollution, contaminated former industrial sites, traffic, unemployment, lack of competitiveness, poverty, demographic changes, and so on)", but it is also "the place where the future is built: universities, research centers, and so on" [4].

Moreover, urban quality of life is also increasingly being seen as an essential element for the development of cities. Florida [14] argues that people are more important than firms in an urban economic context, and that right urban policies should tend to attract "creative" people by improving the conditions in which they live. As Trip [30, p. 502] sums it, "crucial for this is quality of place: an attractive, diverse and tolerant urban environment is being increasingly recognized as a key factor in urban competitiveness". These reflections present new challenges for urban management, as resources being limited, "policy-makers need to find the most efficient way of distributing them in line with the needs and priorities of people" [31, p. 361-362].

However, despite the growing interest and work on the field of quality of life, consistent tools for measuring and improving the wellbeing of citizens are not available. As noted by Lambiri [21, p. 20], the "use of theoretically consistent statistical and econometric methods" for exploring quality of life is an issue that needs "to be addressed more deeply both theoretically and empirically in the economic and urban economic literature".

The main aim of this paper is to allow the operationalization of the quality of life concept, in a way that it becomes possible to 
measure the impact of local policies in the urban quality of life, as well as to compare cities between them. For this purpose, we applied data envelopment analysis (DEA) to evaluate the performance of cities in what concerns the promotion of quality of life, given the economic condition of the country, thus elaborating a model of intervention that allows the identification of urban best practices. DEA was also used to construct a ranking of cities, given their level of quality of life, through the construction of a composite indicator.

\section{Understanding quality of life}

The first step to understand the large academic work on quality of life, mainly in its urban aspect, is given by the identification of the key-concepts, the dimensions and measurement methods more often used. According to Pacione [25, p. 1], it has been central to academic research the effort to examine the "multidimensional aspects of urban environmental quality and human wellbeing".

\subsection{The concept}

It is obvious from a first analysis of the scientific discussion in this field that it is difficult to define the concept of quality of life, since it has not yet stabilized in the literature. There is no agreement on quality of life, in terminology nor in construction methods or the criteria that comprise quality of life [24]. While quality of life is, in some approaches such as the one proposed by Mitchell et al. [24], a set of factors such as health, physical environment, natural resources, personal development and safety, other researchers present economics "as one of the three major pillars of quality" [19, p. 9]. Efforts to reach a consensual definition did not succeed and therefore "the absence of a generally accepted framework for quality of life research is acknowledged as an obstacle to progress" [25, p. 2].

Although these previous references allow us to reach a quantitative concept, some authors defend quality of life as a mainly subjective concept. This is the case of the definition proposed by the WHO-QOL group in 1993, which argues that quality of life is "an individual's perception of his/her position in life in the context of the culture and value systems in which he/ she lives in relation to his/her goals, expectations, standards and concerns" [19, p. 7]. Some authors have presented quality of life as life satisfaction and others as the degree to which a person enjoys the possibilities of his/her life" [19]. Both approaches, quantitative and qualitative, have been fruitful to research and looking at the quality of life in the specific context of cities presents an opportunity to explore the relationship between objective and subjective dimensions, as noted by Marans [23, p. 74].

For the purpose of this paper, we can adopt the definition of quality of life proposed by the Australian project Social Benchmarks and Indicators for Victoria [28, p. 39]: "The overall level of wellbeing and fulfillment that people enjoy from a combination of their social, economic and community environment and their physical and material conditions".

\subsection{The dimensions of quality of life}

When going from the concept to a real-world analysis, we realize that the complexity of quality of life is expressed in the diverse dimensions that are chosen by researchers. This is not only an academic problem, but also a political one. Policy-makers are also interested in defining the factors of satisfaction that their policies may alter, driving researchers to study in what way satisfaction is influenced by various objective conditions. And it should not be forgotten that "quality of life is increasingly considered a crucial element of urban competitiveness and growth" [21, p. 4], thus explaining the interest of urban planners and decisors.

Direct approaches to quality of life assessment differed from place to place. Some proposals from New Zealand [5] have specified several dimensions, including demography, knowledge and skills, health, safety, housing, social connectedness, civil and political rights, economic standard of living, economic development, the natural environment and the built environment. In Canada, in the city of Winnipeg, five dimensions were chosen to express the factors that influence quality of life: individual wellbeing, urban economy, urban environment, community assets, community leadership and pride [18]. In the Urban Audit program (www.urbanaudit.org) - the study of quality of life promoted by the European Union - the definition of the relevant dimensions to evaluate quality of life has been subject to an intensive research effort, resulting in the specification of nine dimensions: demography, social aspects, economic aspects, civic involvement, training and education, environment, transport and travel, culture and leisure, innovation and technology.

From the analysis of these references it is possible to realize that a sort of central core for research has been constructed: the studies undertaken have been focused in a similar set of equivalent dimensions, such as health and economics, that may then be enlarged to a wider conception that reveals a different understanding of quality of life. For example, it can be more focused on individual or collective life of the population (such as the New Zealand study and the Urban Audit, in a certain way) or more directed to urban aspects of space enjoyment.

\subsection{Measuring quality of life}

For each of the dimensions considered in the existing studies, researchers have selected different variables, combined in proper indicators. There is a general consensus in the literature that objective and subjective indicators are needed in the study of the person-environment relationship.

In the literature, the selection of indicators is normally the outcome of researchers reasoning or expert group meetings. This is the case of the Urban Audit project that joins a think tank to evaluate the pertinence of the indicators selected and the need to add or withdraw indicators.

Some studies using a set of indicators have measured quality of life in several cities of different regions of the world, showing that the effort to measure is viable. Some universities have done research in this field and a variety of models have been developed in a non-coordinated and independent way. Some projects, based on a variety of methods, have been set up following the initiative of local governments or cities authorities, such as the "Local Government Act 2000" project of New Zealand that evaluates the quality of life, initially in the eight biggest cities and nowadays in the twelve biggest cities [5]. Another work to be considered is the Australian project Social Benchmarks and Indicators for Victoria [28]. When we consider larger regions, such as Europe, we realize that the effort to coordinate is incipient, but some relevant experiences can already be pointed out, as the Urban Audit project.

One of the perspectives of work adopted in this field is the development of measurement methods such as "the construction of indices through a system of indicators, with the aim to capture as precisely as possible the multidimensionality of the concept" $[21$, p. 20]. Thus, some of the issues that characterize quality of 
life, or other factors influencing the wellbeing of the citizens, such as development, are presented through indices or meta-indices. Some of these indices correspond to concepts that are consolidated in literature and are used in a generalized way.

This is the case for the Human Development Index (HDI), of the United Nations Program for Development, which is a universal and stabilized concept. It embodies wealth, health and education as core factors, allowing a comparison between countries and regions, thus enabling the assessment of the effectiveness of national and regional governmental policies [32]. The HDI is based on three indicators: longevity, measured by life expectancy at birth; educational level, measured by a combination of adult literacy (with two-third weighting) with the combined primary, secondary and tertiary gross enrolment ratio (with one-third weighting); and standard of living, measured by the gross domestic product (GDP) per capita (PPP US dollars). From these indicators it can be obtained a synthetic index. The HDI is the simple mean (arithmetic) of the life expectancy indicators, the educational level indicators and the real adjusted GDP per capita indicators.

There are other composite indices in literature, such as the Environmental Sustainability Index of the World Economic Forum or the Prescott-Allen's Wellbeing Index, that have also been used to allow comparison between countries or regions. Another example of an index of quality of life is the Mercer's study, based on detailed assessments and evaluations of 39 key quality of living determinants.

Evidently, the construction of the composite indices, in which sub-indicators are aggregated into one number, involves stages that need subjective judgment: the selection of sub-indicators, the treatment of missing values, the choice of the aggregation model, or the weights of the sub-indices. As a result, the construction of composite indices is often controversial. For example, in the case of the HDI there is disagreement about the indicators included in the index, how to weight the scores within each dimensions, and how to aggregate the weighted scores over the different dimensions. Despotis [12], for instance, focused on the method of weighting/aggregation of different components and proposed the use of data envelopment analysis (DEA) to reduce the inherent subjectivity associated to the specification of weights. As stated by Despotis [12], the advantage of the new measure proposed is that the weights result from an optimizing process, based on linear programming, so they are less arbitrary and contestable. This approach reflects the fact that it is possible to construct indices using several different methods applied to the same original data, all resulting in different rankings of the units assessed. This is a consequence of "the inherent subjectivity in the choice firstly of indicators, and secondly of the final weights of every indicator used" for the construction of the final composite index [21, p. 20].

DEA is then a versatile approach to performance assessment that can be applied to several areas, in which we include development and quality of life policies. For instance, Gorman and Ruggiero [15] applied this methodology to evaluate state police performance. Despotis [12], as referred previously, applied DEA to the HDI, redesigning the assessment of countries development. Bougnol [6] also demonstrated the advantage of using DEA models for ranking the world's nations using the HDI indicators. Zhu [35] illustrated the potential of this method in an urban context, presenting a study on the economic performance of Chinese cities. To our knowledge, DEA has not yet been applied to the management of urban quality of life, but it presents several advantages, namely in terms of low information requirements [17] and in the ability of handling multiple inputs and outputs. Thus, it can give fundamental contributions to help local policy-makers to achieve better performances in that matter.
This paper develops a procedure to evaluate urban quality of life using a composite performance indicator. The method of weighting for the indicators will be based on DEA, as it looks for the endogenous weights, and thus reduces subjectivity. By its very nature, a summary index is bound to conceal important aspects of the complex phenomenon represented by the indicator. However, we believe it is an essential tool to enable direct comparisons between the quality of life of cities. It also allows the implementation of benchmarking procedures, leading to the identification of best practices that can be spread across cities.

\section{The Urban Audit Project and its city profile}

The Urban Audit Project is a major program that gathers information about cities, in a systematic and exhaustive way, being the most important initiative in the area in Europe. This study aims to provide significant and comprehensive data to characterize European cities, and guide the definition of public European policies. The data collected for several urban aspects is classified in distinct variables, stressing disparities between cities, which is useful, and even crucial, to political decision-making. However, to the best of our knowledge, up to this point there has not been an attempt to create synthetic indicators, or explore the relationships between different variables from the Urban Audit [13]. Thus, we have chosen to use the data of Urban Audit to develop a new model for the evaluation of cities performance, based on the construction of a composite indicator.

The Urban Audit Project is a contribution to the growing need to determine and intervene in the quality of life of European cities. The Urban Audit was included in the core business of Eurostat in the end of 2004, and allows a comparison between cities classified according to their position in Europe (central-peripheral-south). The data is structured in nine dimensions (demography, social aspects, economic aspects, civic involvement, training and education, environment, transport and travel, culture and leisure, innovation and technology). Each dimension includes several variables, totalizing 336 variables. National statistic institutes constitute the main data source used by the Urban Audit.

This project started as a pilot study, in June 1999. At that time, the Commission conducted a data collection of comparable indicators in European cities, where 450 variables were collected for the 58 largest cities. The first full-scale data collection effort of this project took place in 2003, following an evaluation of this pilot project. It led to a more focused list of variables and a significant expansion of participating cities, covering 258 cities in the EU27. A new round of Urban Audit data collection started in May 2006, including information of 284 cities ( 189 belonging to the Europe of 15, 69 to the new 12 Member-States and 26 to Turkey).

Besides this information, and to evaluate the subjective perception of quality of life by citizens, the Urban Audit has a set of indicators that complement the objective data. This data is obtained from surveys applied to large random samples of inhabitants of the cities studied. Due to the cost of collecting this data, only 31 cities were surveyed in 2003, but in 2006 and 2009 the sample increased to 75 cities.

For each city, the Urban Audit program establishes a city profile that includes a subset of aspects of quality of life organized in seven of the nine dimensions considered in the study, i.e., demography, social aspects, economic aspects, civic involvement, training and education, environment, transport and travel. Culture and leisure, as well as innovation and technology, are not part of the actual city profile designed by the Urban Audit, although the methodological support for this decision is not clear in the presentation of the concept (see www.urbanaudit.org). 
Table 1

Outputs considered in the DEA model.

\begin{tabular}{|c|c|}
\hline Demography & $\begin{array}{l}\text { (01) Growth of population over } 2 \text { years in percentage of resident population } \\
\text { (O2) Percentage of households with more than one person } \\
\text { (O3) Percentage of households that are not lone-parent household }\end{array}$ \\
\hline Social aspects & $\begin{array}{l}\text { (O4) Average price for an apartment (in euros per } \mathrm{m}^{2} \text { ) } \\
\text { (O5) Average price for a house (in euros per } \mathrm{m}^{2} \text { ) } \\
\text { (O6) Percentage of households living in owned dwellings } \\
\text { (O7) Percentage of households not living in social housing } \\
\text { (O8) Average area of living accommodation ( } \mathrm{m} 2 \text { per person) } \\
\text { (O9) Life expectancy at birth (years) } \\
\text { (010) Population per recorded crime }\end{array}$ \\
\hline Economic aspects & $\begin{array}{l}\text { (011) Employment rate (\%) } \\
\text { (012) Female employment rate (\%) } \\
\text { (013) Activity rate (\%) } \\
\text { (014) Median disposable annual household income (in euros) } \\
\text { (015) \% of households receiving more than half the national average household income }\end{array}$ \\
\hline Civic involvement & (016) Percentage of registered electorate voting in city elections \\
\hline Training and education & $\begin{array}{l}\text { (017) Percentage of children aged } 0-4 \text { in day care } \\
\text { (018) Percentage of resident population with secondary education } \\
\text { (019) Percentage of resident population with tertiary education }\end{array}$ \\
\hline Environment & $\begin{array}{l}\text { (O20) No. of days with ozone concentrations below } 120 \mu \mathrm{g} / \mathrm{m}^{3} \text { (per year) } \\
(\mathrm{O} 21) \text { No. of days with particulate matter concentrations below } 50 \mu \mathrm{g} / \mathrm{m}^{3} \text { (per year) } \\
\text { (O22) Percentage of solid waste processed by landfill } \\
\text { (O23) Green space to which public has access }\left(\mathrm{m}^{2} \text { per } 10,000 \text { inhabitants) }\right.\end{array}$ \\
\hline Transport and travel & $\begin{array}{l}\text { (O24) Percentage of journeys to work not done by car } \\
\text { (O25) Average time saved in journeys to work in relation to the time reference of } 75 \mathrm{~min}\end{array}$ \\
\hline Information society & (O26) Percentage of households with a PC \\
\hline Culture and recreation & $\begin{array}{l}\text { (O27) Cinema attendance (per year per capita) } \\
\text { (O28) No. of museum visitors (per year per capita) } \\
\text { (O29) No. of theatre seats per } 1000 \text { inhabitants }\end{array}$ \\
\hline
\end{tabular}

\section{The methodology}

This paper has two main purposes: to evaluate quality of life through the development of a composite indicator, and to provide a tool to promote improvements in quality of life. Concerning the second objective, this paper intends to evaluate to what extent policy-makers of cities transform the wealth of their country, measured by the GDP per capita, in quality of life. Data envelopment analysis (DEA) was the technique selected to construct the composite indicator of quality of life and, in the next step, to establish a model for the evaluation of local policies.

\subsection{Inputs and outputs of the DEA model}

The first step of the DEA assessment is to determine the indicators (outputs) that will be used to assess city performance. Having said before that this work uses the data provided by Urban Audit, the obvious difficulty to deal with 336 variables stands up. In order to have a more operational and reduced set of data, we decided to used the city profile provided by Urban Audit, as it is already the result of a field work experience, and the indicators reflect the central core of quality of life as it is understood by the Urban Audit project. However, the concept of city profile used by this European project does not include the components of culture and leisure and innovation and technology. As we believe these dimensions are important for quality of life assessment, we have also included these two dimensions in this study. As Picavet [26, p. 1128] puts it, reasoning on the ethics of operational research, "it is especially important to select goals and parameters of interest in a relevant way if the expert if to offer proper guidance to a human community". We then have a city profile in which all the nine dimensions of quality of life used in the Urban Audit are considered.
Following this approach, we looked at the indicators chosen by the City Profile for each dimension. For the purpose of our work, we chose only the ones we considered to be indicative of quality of life (e.g. percentage of households living in owned dwellings), such that higher values represent better quality of life. We excluded indicators that may suggest an improvement in quality of life up to a critical point, but whose contribution may be doubtful beyond that threshold, such as population density or immigrants as a percentage of total population. Indicators which were negatively related to quality of life (e.g., percentage of households receiving less than half of the national average household income) were transformed into positive aspects of quality of life (e.g. percentage of households receiving more than half of the national average household income), by using the related/opposite/complementary indicator, to satisfy the basic DEA assumption that more output is better. Thus, we have built a new concept of city profile, based on a different selection of indicators that, clearly and objectively, reveals quality of life.

The result is a total of 29 quality of life indicators, which correspond to the outputs of the DEA model. The outputs are distributed by the nine dimensions, as shown in Table 1 .

Being a vast and relatively recent project, Urban Audit suffers a major limitation related to data unavailability for several variables in many cities. Even though there was a data collection in 2006, this work deals with the 2003 campaign because it followed national census in a significant number of countries, thus relying on more precise data. Data from 2006 has more missing values and relies more on estimations, losing in precision what it gains in timeliness. Before applying DEA to our sample and in order to avoid bias in the analysis that could be introduced by missing data, we decided to include in our study only the cities with a minimum of 10 valid observations in the 29 outputs considered. A total of 50 cities were eliminated from the original database of the Urban Audit program. 
In the other cities, missing values were substituted by the minimum value observed in the output considered. A consensual way to deal with missing values has not yet been reached in the DEA literature. Several solutions to model missing values have been discussed, such as the proposal of Kuosmanen [20] to replace missing outputs by zero, or the approach of Smirlis et al. [29] that suggest the replacement of missing values by approximations in the form of interval in which the unknown missing values are likely to belong. For the purpose of this work, we have chosen to replace the missing observations with the minimum value observed in the database for that variable. This ensures that cities with no data available are not benefited in the performance assessment in relation to their counterparts. The missing values were not replaced by zero because this would hinder the assessment of cities with missing values in the variables that represent a dimension (e.g., output 26 in the information society dimension or output 16 in the civil involvement dimension). In a total of 5974 observations considered to be valid, $32 \%$ resulted from the substitution of missing values. The variables for which the problem of missing data was more acute were outputs 14 and 15 (related to households income), output 22 (percentage of solid waste processed by landfill), and output 26 (percentages of households with a PC).

Finally, we explored the existence of outliers in the sample. It is known that in the presence of outliers, the location of the DEA frontier could be severely affected due to its sensibility to extreme observations. This required the removal of two more cities from the original database. Our sample is then constituted by 206 cities, belonging to 25 countries.

The next step is to define the inputs of our study. In the analysis described in this paper, we first constructed a composite indicator of quality of life, which implies comparing quality of life at the city level only based on the outputs. This is equivalent to assume that all cities have the same input levels, which can be represented in a DEA formulation as a single "dummy" input equal to one for all DMUs.

We used a second approach to respond to a different purpose, which is the ability of local authorities to promote quality of life given the economic condition of the country. To account for the fact that it is not possible to consider all cities in equal circumstances, given the different level of the wealth of the country, we need to put in perspective the fact that cities cannot be compared per se without consideration of national conditions. One possibility would be to introduce national gross domestic product (GDP) per capita of each city as the input, which allows to clearly assess the capacities of local authorities to induce quality of life given the national context in which their cities are implanted. We did not choose an input focused in urban or administrative dimensions such as the budget of local administrations for cities management, as this option would have presented two kinds of problems: there is not a single model in the European cities for governance separation between local and central administrations, which has obvious implications on the revenues that each city can expect for its budgetary expenses; secondly, not all the outputs considered in the city profile depend exclusively in cities characteristics. Therefore, a value that clearly can be compared at an European level and that can be expected to induce quality of life is GDP per capita. GDP per capita has previously been used in the literature as an input of a DEA assessment, such as in Wu et al. [34]. In our study, this was the only input variable used to evaluate the cities efficiency in the promotion of quality of life.

\subsection{Composite indicator of quality of life}

DEA, first introduced by Charnes et al. [8], is a linear programming technique for comparing the efficiency of a relatively homogeneous set of organizational decision making units, such as schools [27], banks [2,22,7] or business firms, in their use of multiple resources (inputs) to produce multiple outcomes (outputs). DEA derives a single summary measure of efficiency for each unit, which is based on a comparison with the other units in the sample. This assessment is relative since the units are compared between each other. DEA identifies a subset of efficient units, considered as examples of best practices or benchmarks. For the inefficient units, the magnitude of their efficiency is derived by the distance to the frontier constructed from the benchmarks. The comparison with the benchmarks also allows determining the input and output targets corresponding to efficient operation.

Although the DEA models estimate efficiency as ratios of outputs and outputs, it is also possible to use DEA to construct composite indicators $(\mathrm{CI})$, following the research line initiated by Cherchye et al. [9]. The main difference between a traditional DEA efficiency analysis and the construction of a $\mathrm{CI}$ is that the latter only looks at achievements, without taking into account the resources used. The underlying idea of using a DEA model to obtain a composite indicator of quality of life for a city is to merge the quality of life indicators (i.e., the outputs of the DEA model), in a single summary measure of performance. The advantage of DEA in this case is to allow each city to select its own weighting system for the evaluation of performance, recurring to optimization procedures that emphasize the city's strengths. The linear programming model for deriving the composite indicator of each city is shown in (1). As stated in Cherchye et al. [9, p. 121] model (1) is equivalent to the original DEA input oriented model of Charnes et al. [8], with all indicators considered as outputs and a "dummy input" equal to one for all DMUs

$$
\begin{aligned}
& C I_{0}=\max \sum_{r=1}^{s} u_{r} y_{r j_{0}} \\
& \text { s.t. } \\
& \sum_{r=1}^{s} u_{r} y_{r j} \leq 1, \quad j=1, \ldots, n, \\
& u_{r} \geq 0, \quad r=1, \ldots, s
\end{aligned}
$$

In model (1) $y_{r j}(r=1, \ldots, s)$ corresponds to the value of the output indicator $r$ in city $j(j=1, \ldots, n)$, with higher values corresponding to better performance. The main difficulty associated with the estimation of an aggregate performance indicator is the selection of appropriate weights. Model (1) overcomes this problem recurring to optimization procedures, which determine the weights that give the highest possible score for each unit assessed, keeping the scores of all other units less than or equal to one when evaluated with similar weights. The weights $u_{r}$, $(r=1, \ldots, s)$ are thus the variables of model (1). It should be noted that these weights can be different for every unit $j_{0}$ being analyzed. Each unit final weights are assigned so it will attain the best possible score when compared to peer units that are temporarily assigned the same set of weights. If the city assessed does not obtain an efficiency score of one using this procedure, this means that its peers perform better even when all the weights are set to maximize the score of the focus city. So, this is not only a sound justification for the weights assigned, but also no city can complain that its score would have been better if a different set of weights were used. The composite indicator score $C I_{0}$ of city $j_{0}$ is between 0 (worst) and 1 (best). A composite indicator equal to one signals best observed performance, and lower values signal potential for improvement, which may be achieved by following the best practices observed in peer cities.

Although model (1) is very powerful to derive an overall, objective, summary measure of performance, the non-negativity 
restrictions on the weights $\left(u_{r}\right)$ allow for extreme scenarios. The flexibility in the choice of weights, which is strength of a DEA analysis, may also be a weakness, as it allows some indicators to be assigned a zero weight, meaning that these factors are in fact ignored in the performance assessment. If a city has a particularly high value for an output indicator, this city may dominate the other cities in this particular dimension, and thus obtain a score equal to one simply because all other indicators are given a weight equal to zero. Furthermore, if the number of outputs is large compared to the number of units assessed, this may lead to a situation with a large number of cities achieving a score of one, without allowing for discrimination between good and bad performers. It is possible to overcome this limitation by introducing additional constraints on the weights, to discriminate the real best-performing cities from the others that only achieve a score of one due to a judicious choice of weights. The issue of imposing weight restrictions in the DEA model attracted considerable attention in the DEA literature (see [1] for a review). As suggested by Cherchye et al. [10], restrictions to virtual outputs, corresponding to the dimensions of quality of life specified in our model, are particularly interesting in this context of estimation of composite indicators, as these do not depend on the measurement units and directly reveal how the respective dimension contributes to the performance score.

The first study to apply restrictions to virtual weights in DEA models was that of Wong and Beasley [33]. These restrictions limit the proportion of the total virtual output of DMU $j$ devoted to output $r$, i.e., the importance attached to that output. Such restrictions, which are DMU specific, can be expressed as

$\alpha_{r} \leq \frac{u_{r} y_{r j}}{\sum_{r=1}^{s} u_{r} y_{r j}} \leq \beta_{r}, \quad r=1, \ldots, s, \quad j=1, \ldots, n$

In expression (2), the total virtual output of DMU $j\left(\sum_{r=1}^{s} u_{r} y_{r j}\right)$ is included in the denominator as a standardization mechanism, that facilitates the assignment of the values for $\alpha_{r}$ and $\beta_{r}$, which should be between 0 and 1 . These values are intended to reflect prior views of experts on the relative importance of the individual outputs.

Wong and Beasley [33] suggest a few alternatives for implementing restrictions on virtual weights. The alternative that is more in line with the logic of traditional DEA models is to add the restrictions in respect to all DMUs being compared. This implies that the weights chosen to evaluate DMU $j$ must be such that its proportional constraint is satisfied, and when the same set of weights is applied to all other DMUs, their proportional constraints must also be satisfied. However, this approach may become computationally expensive, and leads to infeasibilities even when the bounds are loosely specified. We used this approach to derive the composite indicator of urban quality of life, but imposed minimum assumptions in relation to the relative importance of the outputs. In order to reduce the need for detailed assumptions/choices, we specified the weight restrictions on a more aggregate level. Specifically, as the outputs were classified in nine dimensions (see Table 1 ), we specified the restrictions with respect to the percentage share of each dimension. In order to avoid having dimensions that do not contribute to the construction of the composite indicator, we specified a virtual weight for all dimensions greater or equal to $1.5 \%$ as follows:

$\frac{\sum_{r \in D_{z}} u_{r} y_{r j}}{\sum_{r=1}^{S} u_{r} y_{r j}} \geq 1.5 \%, \quad z=1, \ldots, 9, \quad j=1, \ldots, n$

The advantage of specifying a low value for the parameter $\alpha_{z}$ is to allow for flexibility in the selection of the weights, such that each city will still be allowed to show itself in a favorable light. In addition, from the composition of the virtual weights at the optimal solution for the DMU under assessment, it will be possible to identify the strengths and weaknesses of each city. This procedure also allows improving significantly the discrimination power of the DEA model. Using model (1) without weight restrictions to build a composite performance indicator for the sample of 206 European cities, only 14 cities were classified as inefficient, with the remaining 192 receiving a score equal to 1 . Conversely, adding the restrictions in (3) to model (1), the discrimination power of the model increased dramatically with only four cities classified as fully efficient. The main limitation of the restrictions on the virtual weights is that they represent indirect absolute bounds on the DEA weights, so they are sensitive to model orientation and may lead to infeasible solutions (for further details see [1]).

The assessment of QoL using model (1) with the weight restrictions (3) provides an evaluation of cities performance where diversity is accounted for, i.e., the cities that excel at given dimensions are credited for that, and their overall score reflects their good performance in given aspects.

The next step of our analysis consisted on deriving an overall ranking of quality of life at the city and country levels. To be able to compare cities and countries performance, the assessment should be done on the same basis, this meaning that it would be desirable to evaluate all cities with a common set of weights. Subsequently, the countries performance can be obtained as the average performance of their cities. To implement this approach, we used the goal programming model shown in (4). This model finds a set of weights that minimizes the deviations $\left(z_{j}\right)$ in relation to the composite indicator score obtained in the previous stage of the assessment, based on model (1) with restrictions (3)

$$
\begin{array}{ll}
\operatorname{Min} \sum_{r=1}^{s} z_{j} & \\
\text { s.t. } & \\
\sum_{r=1}^{s} u_{r} y_{r j}+z_{j}=C I_{0}, & j=1, \ldots, n, \\
u_{r} \geq 0, & r=1, \ldots, s, \\
z_{j} \geq 0, & j=1, \ldots, n
\end{array}
$$

\subsection{Evaluation of city efficiency in the promotion of quality of life}

For the analysis of cities efficiency in terms of their ability to promote quality of life given the economic condition of the country, we defined the GDP per capita as the input of the DEA model. The model developed for this purpose is intended to suggest directions for improvement that may be followed by local administrators. Since quality of life is perceived by citizens as a multidimensional concept, it is important that the improvements suggested lead to a balanced equilibrium between all quality of life dimensions, without focusing only on a subset of dimensions in detriment of others. Therefore, we decided to use tighter weight restrictions when going from a model of performance assessment (as described in the previous section) to a model of performance management (as described in this section), such that all dimensions would be reflected in the efficiency score. Since there is no agreement in the literature concerning the relative importance of the dimensions, we imposed equal virtual weights to each of the nine dimensions of quality of life. In this case, the only flexibility allowed in the DEA model concerned the selection of weights for the indicators within each dimension.

However, with this weight specification, if the restrictions were imposed to all DMUs being assessed, the DEA model would 
become infeasible. Therefore, we modified the implementation of the weight restrictions, and only added restrictions to the DMU being assessed, leaving free the relative virtual values of the other DMUs, as suggested by Wong and Beasley [33]. The model used is shown in (5). This model corresponds to an output oriented model, assuming variable returns to scale, as formulated first by Banker et al. [3]. The use of this formulation is required because the outputs specified for the assessment of cities' quality of life correspond to ratios or percentages. As shown by Hollingsworth and Smith [16, p. 735], the use of the original DEA model of Charnes et al. [8] in these circumstances would produce "perverse and technically incorrect results". The optimal solution of the linear programming model (5) enables calculating the efficiency score of city $j_{0}$, which is given by $1 / h_{0}$. This score is between 0 (worst) and 1 (best)

$h_{0}=\operatorname{Min} \sum_{i=1}^{m} v_{i} x_{i j_{0}}+\varpi$

S.t

$\sum_{r=1}^{s} u_{r} y_{r j_{0}}=1$

$-\sum_{r=1}^{s} u_{r} y_{r j}+\sum_{i=1}^{m} v_{i} x_{i j}+\varpi \geq 0, \quad j=1, \ldots, n$,

$\sum_{r \in D z} u_{r} y_{r j_{0}}=\frac{1}{9}$

$z=1, \ldots, 9$,

$u_{r} \geq 0$,

$v_{i} \geq 0$,

$r=1, \ldots, S$,

$i=1, \ldots, m$,

$\varpi$ is free

In this model, as the virtual weight restriction is only imposed to the DMU being assessed, the denominator of the general formulation of this type of weight restrictions is always equal to one, so the specification of the virtual restrictions reduces to expression (5a).

The envelopment formulation of the DEA model (5) is shown in (6). This formulation was used to facilitate the computation of the peers, i.e. the cities considered good performers when evaluated with similar weights, and targets for DMU $j_{0}$

$\operatorname{Max} \delta+\frac{1}{9} \sum_{j=1}^{n} \sum_{z=1}^{9} \rho_{z j}$

s.t.

$\sum_{j=1}^{n} \lambda_{j} x_{i j} \leq x_{i j_{0}}, \quad i=1, \ldots, m$,

$\left(\delta+\rho_{z j}\right) y_{r j_{0}}-\sum_{j=1}^{n} \lambda_{j} y_{r j} \leq 0, \quad r \in D z, \quad z=1, \ldots, 9$,

$\sum_{j=1}^{n} \lambda_{j}=1$

$\lambda_{j} \geq 0$ $\forall j$

$\delta$ and $\rho_{\mathrm{zj}}$ are free

Note that using this formulation it is possible that a DMU considered inefficient by model (6) may have as peers DMUs that are also inefficient when evaluated with model (6), although they are deemed to be efficient in an evaluation using the unrestricted DEA model. This is explained by the fact that the peers for the DMU under assessment are not subject to weight restrictions, which are only imposed to the DMU under assessment. In relation to the targets suggested for inefficient DMUs, the weight restrictions allow trade-offs between the output dimensions, such that some output dimensions may be suggested an increase at the expense of a decrease in other output dimensions, leading to a more balanced performance profile of the city.

\section{Results and discussion}

This section describes two different types of results, depending on the model used, following the purpose of the work: either to construct a composite indicator of urban quality of life (with a flexible weight assignment system and with a common set of weights) or to assess the performance of city management, i.e., the ability of cities to transform national wealth (measured by GDP) into quality of life. The second approach enables the proposal of directions for improvement, which can be followed by local administrators.

\subsection{Results for the composite indicator of quality of life}

The composite indicator is a useful tool when the aim is to consider all the DMU in a global perspective, providing an overview of the quality of life of cities. For instance, in the perspective of the European Union, this composite indicator can be useful to identify where intervention is most needed to increase the quality of life of European cities. This analysis allows to value the dimension or dimensions in which the city has a good performance, thus distinguishing the high performers from the rest of group.

The performance assessment using the DEA model described in Section 4.2, with the output indicators subject to weight restrictions for all DMUs, only four cities achieved the maximum score of the composite indicator of quality of life (corresponding to 100\%). The average score for the sample analyzed was $51 \%$. When we look at the cities that are high performers, more specifically to the 20 best cities, listed in Table 2, we can conclude that the majority belongs either to Germany (7), Belgium (3) or Netherlands (3). Although none of these German, Dutch or Belgian cities are the capital of their country, it is also a remarkable fact that the other cities (6) are all capital cities of their country, except Gozo. In the 20 cities with the worst performances, the presence of Eastern countries stands out, with cities belonging mainly to Romania (7), Italy (6) and Poland (3). These cities demand more attention when a global perspective of European cohesion is at stake.

Table 2

Best and worst cities according to the Quality of Life assessment.

\begin{tabular}{|c|c|c|c|}
\hline \multicolumn{2}{|c|}{ Best QoL (top 20) } & \multicolumn{2}{|c|}{ Worst QoL (bottom 20) } \\
\hline Countries & Cities & Countries & Cities \\
\hline \multirow[t]{7}{*}{ Germany } & Dresden & Romania & Targu Mures \\
\hline & Weimar & & Sibiu \\
\hline & Freiburg im Breisgau & & Craiova \\
\hline & Frankfurt am Main & & Timisoara \\
\hline & Darmstadt & & Bucaresti \\
\hline & Nurnberg & & Arad \\
\hline & Dortmund & & Oradea \\
\hline \multirow[t]{3}{*}{ Netherlands } & Groningen & Italy & Bari \\
\hline & Arnhem & & Napoli \\
\hline & Gravenhage & & Trieste \\
\hline \multirow[t]{3}{*}{ Belgium } & Brugge & & Palermo \\
\hline & Antwerpen & & Reggio di Calabria \\
\hline & Liège & & Taranto \\
\hline Luxembourg & Luxembourg & Poland & Suwalki \\
\hline Portugal & Lisboa & & Katowice \\
\hline Finland & Helsinki & & Zory \\
\hline Sweden & Stockholm & UK & Lincoln \\
\hline Malta & Gozo & & Wrexham \\
\hline Austria & Wien & Germany & Moers \\
\hline Cyprus & Lefkosia & Bulgaria & Burgas \\
\hline
\end{tabular}




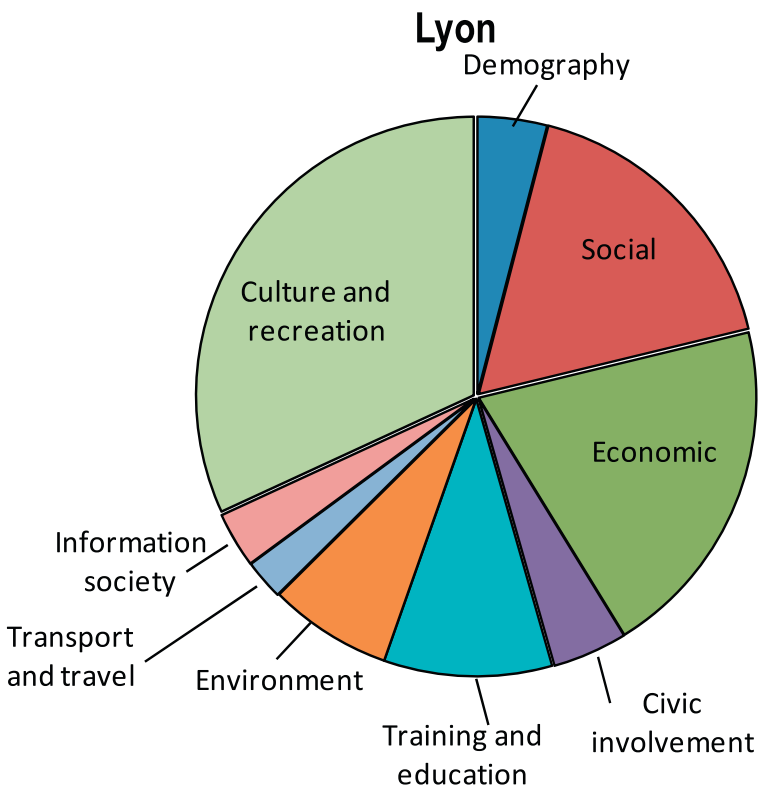

Fig. 1. Contributions of different dimensions for the composite indicator of QoL for Lyon.

Table 3

Relative importance of the dimensions with common weights.

\begin{tabular}{ll}
\hline Dimensions & Virtual weights \\
\hline Demography & 0.02 \\
Social & 0.06 \\
Economic & 0.01 \\
Civic involvement & 0.02 \\
Training and education & 0.05 \\
Environment & 0.02 \\
Transport and travel & 0.04 \\
Information society & 0.06 \\
Culture and recreation & 0.15 \\
\hline
\end{tabular}

This methodology can be particularly interesting for the analysis of the strengths and weaknesses of the cities. The optimization procedure underlying the derivation of the composite indicator using DEA selects the weights that show the city under assessment in the best possible light. Therefore, the best dimensions are given higher virtual weights, and the others are given less weight, with the minimum virtual weight restricted to be $1.5 \%$ for all dimensions. Fig. 1 shows the pie chart characterizing the performance in Lyon, the city selected for illustration. This city has a score of $46 \%$ for the QoL indicator. Lyon shows relatively well balanced performance, with culture and recreation, economic and social corresponding to the best dimensions, followed by training and education, and environmental aspects.

The analysis can go further to make direct comparisons among cities more fair and balanced. This requires the selection of a common set of weights to evaluate all cities, which was done using the goal programming model described in Section 4.2. Table 3 shows the dimensions that were given higher weights using the goal programming model. To make the interpretation of the relative importance of the dimensions easier, Table 3 reports the virtual weights that represent the common framework used for the comparisons (i.e., the variables $u_{r}$ of model (4) multiplied the average value of the indicator $y_{r}$ for all cities analyzed).

The values reported in Table 3 show that in the evaluation of QoL with a common set of weights, the most important dimension is culture and recreation. Social aspects and information society follow, as well as training and education and transport and travel dimensions. Demography, economic aspects, civic involvement and environment are the less expressive dimensions.

Using a common set of weights in the assessment of QoL, cities can be put side by side and compared. This also enables the construction of a ranking of cities. The cities in the 90th and 10th percentile of the ranking are presented in Table 4. An examination of these results shows that, in the top of the list, German cities are highly represented (10), followed by capital cities (6) and urban centers from Netherlands (3) and Belgium (2). At the bottom of the list, Romanian (7), Italian (6), Bulgarian (4) and Polish (2) cities stand out.

Concerning direct comparisons of cities when equal weights are assigned to the dimensions, we illustrate how the profiles of

Table 4

Ranking of cities QoL based on common weights.

\begin{tabular}{|c|c|c|c|}
\hline \multicolumn{2}{|l|}{ Top of the ranking } & \multicolumn{2}{|l|}{ Bottom of the ranking } \\
\hline City & QoL indicator (\%) & City & QoL indicator (\%) \\
\hline Helsinki (Finland) & 100 & Varna (Bulgaria) & 23 \\
\hline Weimar (Germany) & 100 & Targu Mures (Romania) & 22 \\
\hline Luxembourg (Luxembourg) & 100 & Timisoara (Romania) & 22 \\
\hline Dresden (Germany) & 99 & Napoli (Italy) & 22 \\
\hline Frankfurt am Main (Germany) & 91 & Ruse (Bulgaria) & 22 \\
\hline Groningen (Netherlands) & 90 & Burgas (Bulgaria) & 21 \\
\hline Freiburg im Breisgau (Germany) & 88 & Plovdiv (Bulgaria) & 21 \\
\hline Nurnberg (Germany) & 83 & Arad (Romania) & 21 \\
\hline Wien (Austria) & 80 & Suwalki (Poland) & 21 \\
\hline Dortmund (Germany) & 80 & Craiova (Romania) & 21 \\
\hline Arnhem (Netherlands) & 77 & Bucaresti (Romania) & 21 \\
\hline Antwerpen (Netherlands) & 75 & Liepaja (Latvia) & 21 \\
\hline Liége (Belgium) & 74 & Bari (Italy) & 21 \\
\hline Regensburg (Germany) & 74 & Trieste (Italy) & 21 \\
\hline Lisboa (Portugal) & 74 & Palermo (Italy) & 21 \\
\hline Gravenhage (Netherlands) & 74 & Oradea (Romania) & 21 \\
\hline Stockholm (Sweden) & 73 & Sibiu (Romania) & 20 \\
\hline Karlsruhe (Germany) & 73 & Wrexham (UK) & 20 \\
\hline Darmstadt (Germany) & 72 & Zory (Poland) & 19 \\
\hline Munchen (Germany) & 72 & Taranto (Italy) & 17 \\
\hline Amsterdam (Netherlands) & 71 & Reggio di Calabria (Italy) & 16 \\
\hline
\end{tabular}




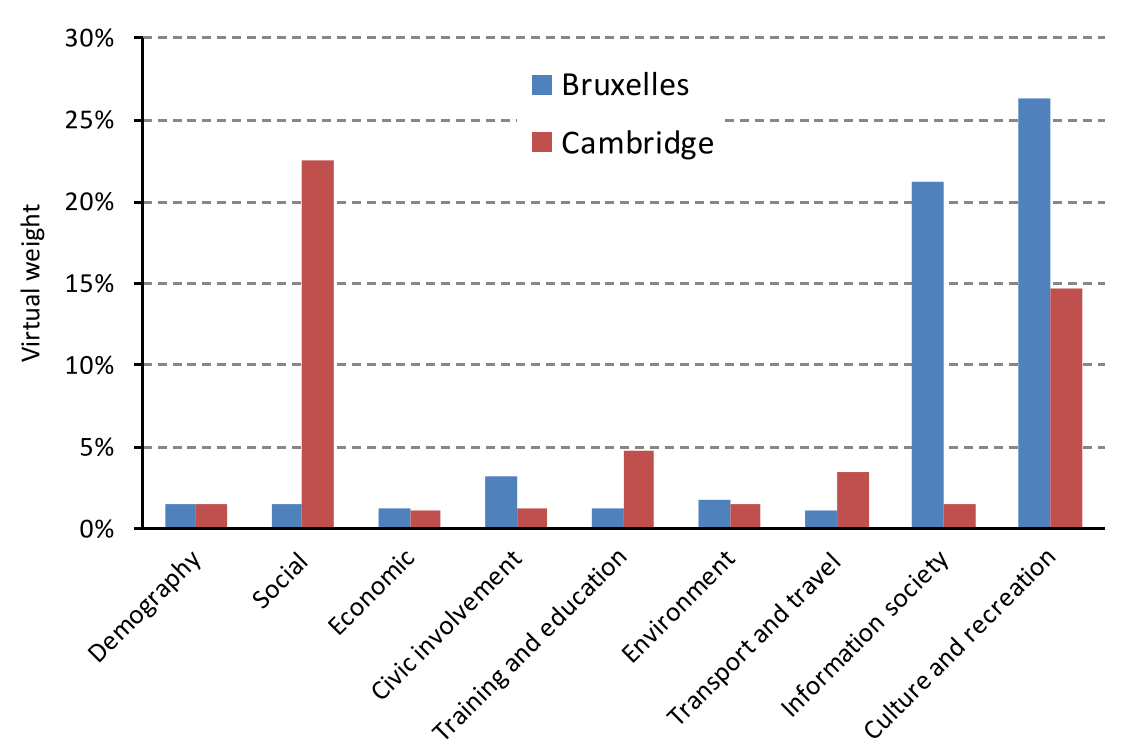

Fig. 2. Contribution of different dimensions for the composite indicator at the city level (with common weights).

Table 5

QoL indicator at the country level (with a common set of weights).

\begin{tabular}{llll}
\hline Country & QoL Indicator & & $\begin{array}{c}\text { No. of cities } \\
\text { assessed }\end{array}$ \\
\cline { 2 - 3 } & Mean (\%) & SD (\%) & \\
\hline Luxembourg & 100 & - & 1 \\
Belgium & 66 & 9 & 6 \\
Netherlands & 63 & 17 & 10 \\
Germany & 62 & 18 & 35 \\
Finland & 60 & 29 & 4 \\
Austria & 59 & 20 & 3 \\
Malta & 54 & 15 & 2 \\
Denmark & 53 & 2 & 4 \\
Cyprus & 52 & - & 1 \\
Estonia & 51 & 0 & 2 \\
Sweden & 51 & 15 & 4 \\
Hungary & 49 & 3 & 4 \\
Slovakia & 48 & 6 & 3 \\
Spain & 44 & 10 & 8 \\
Portugal & 42 & 18 & 5 \\
Czech Rep. & 42 & 5 & 5 \\
Slovenia & 38 & 5 & 2 \\
UK & 37 & 11 & 23 \\
France & 36 & 6 & 26 \\
Lithuania & 30 & 5 & 3 \\
Latvia & 30 & 13 & 22 \\
Poland & 28 & 5 & 18 \\
Italy & 28 & 1 & 8 \\
Romania & 22 & & \\
Bulgaria & 22 & & \\
\hline & & & 2 \\
\hline
\end{tabular}

the cities can be contrasted. Fig. 2 shows the profile of two cities selected for illustration: Bruxelles and Cambridge. These cities have a QoL score of $59 \%$ and $52 \%$, respectively. Note that the sum of the virtual weights shown for all the dimensions of each city is equal to the QoL score. Bruxelles is an example of a city with two strong dimensions (information society and culture and recreation). It also performs better than Cambridge in civic involvement. Cambridge is stronger than Bruxelles in the social aspects, followed by training and education and transport and travel. The performance concerning the other three dimensions (demography, economic and environment) is similar in the two cities.

Finally, the methodology applied to the construction of a composite indicator of city quality of life can have a secondary outcome, leading to a global evaluation for countries, as shown in
Table 5. For each country, the QoL indicator was obtained as the average score of composite indicator of its cities using the common weights. This ranking shows some countries with poor performance in terms of urban quality of life, such as the excommunist countries: Bulgaria (22\%), Romania (22\%) and Poland (28\%). But western countries like France, United Kingdom and Italy have also some weaknesses in their urban quality of life, and present a relatively low average value of the quality of life composite indicator (below 40\%). If we do not consider the country appearing at the top of the list, since it reflects the value of a single city, we find that the countries with best urban practices are all from Old Europe: Belgium (66\%), Netherlands (63\%), Germany (62\%) and Finland (60\%). But these national performances are not uniform: the high standard deviation shows that bad and good practices co-exist in those countries.

Similarly to the comparison of strengths and weaknesses of cities supported by the graphical representation of virtual weights, we can represent the countries profile. Fig. 3 shows an example of the profile for Netherlands and Romania, two countries that rank very differently in our list. Netherlands is strong in the culture and recreation aspect and, to a lesser extent, in the Information Society. Social aspects and Transports and Travel are dimensions where Netherlands also perform relatively well. The major strength of Romania in terms of quality of life is training and education, a dimension very important in the overall score of the country.

5.2. Results for the evaluation of city efficiency in the promotion of quality of life

Applying the DEA model (5) to our sample, with national GDP per capita as the input, 19 cities (listed in Table 6) were considered fully efficient in terms of the achievement of good quality of life standards given the national economic context. In order to discriminate between the most efficient cities, we can use a complementary measure given by the number of times that those cities are peers for others in the DEA assessment. When this measure is considered, Dresden and Helsinki are the cities that are more frequently selected by others as benchmarks.

Looking at the efficient cities listed in Table 6, we realize that Germany (four cities) is highly represented, as well as capital cities (six cities). The presence of cities from Eastern Europe stands out, especially from Bulgaria (but also from Romania, 


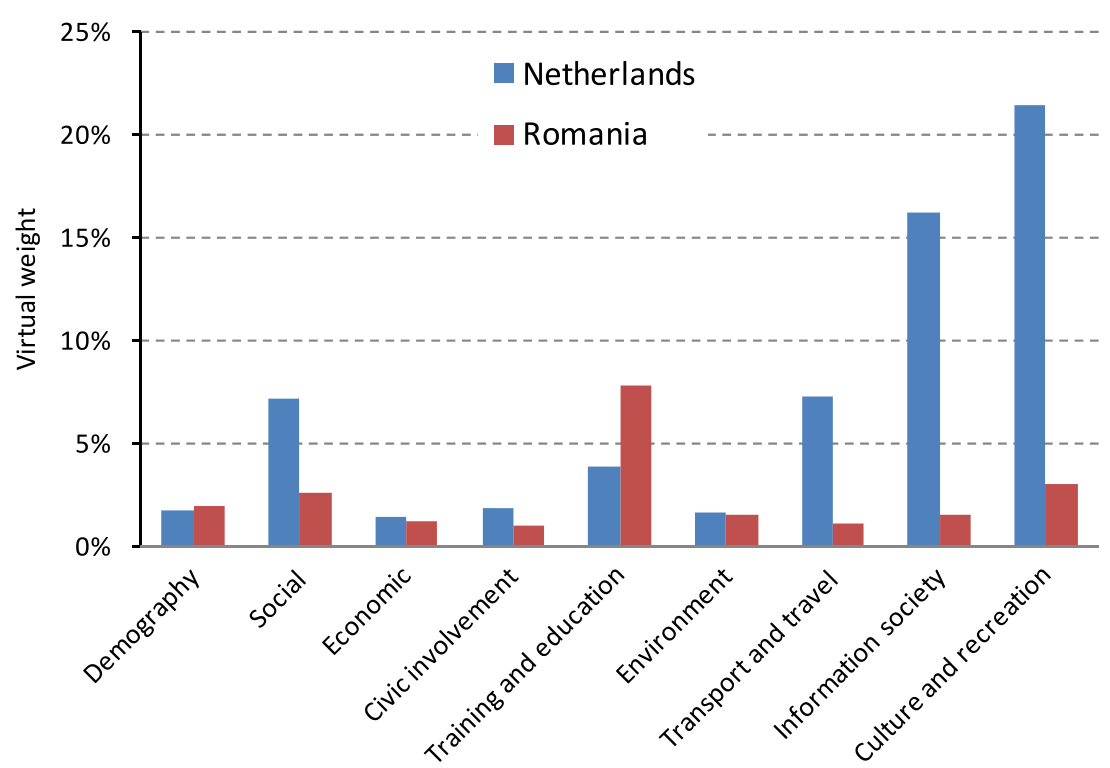

Fig. 3. Contribution of different dimensions for the composite indicator at country level (with common weights).

Table 6

List of cities with the maximum efficiency score in the assessment contextualised by GDP.

\begin{tabular}{ll}
\hline City & No. of times as peers \\
\hline Dresden (Germany) & 74 \\
Helsinki (Finland) & 71 \\
Frankfurt am Main (Germany) & 30 \\
Nitra (Slovakia) & 30 \\
Tartu (Estonia) & 29 \\
Groningen (Netherlands) & 22 \\
Bratislava (Slovakia) & 20 \\
Luxembourg (Luxembourg) & 15 \\
Cluj-Napoca (Romania) & 9 \\
Freiburg im Breisgau (Germany) & 8 \\
Ruse (Bulgaria) & 4 \\
Timisoara (Romania) & 3 \\
Tallinn (Estonia) & 2 \\
Varna (Bulgaria) & 1 \\
Schwerin (Germany) & 0 \\
Wien (Austria) & 0 \\
Plovdiv (Bulgaria) & 0 \\
Burgas (Bulgaria) & 0 \\
Sofia (Bulgaria) & 0
\end{tabular}

Slovakia and Estonia). This does not mean that these cities are the best in terms of quality of life, but that the values of the quality of life indicators are good and well balanced across all dimensions, given the level of GDP.

The cities where intervention may be more in need are shown in Table 7. These correspond to the cities below the 10th percentile, and are mainly from Italy (9) and France (6), with UK, Spain and Germany having two cities each in the bottom of the ranking. We can conclude that these cities, all western urban centers and belonging to old EEC, present low levels of quality of life, given their national economic context (when compared, for example, to Eastern cities).

Using this methodology, we can identify a relevant group of cities with relatively low efficiency scores, thus presenting an opportunity for the development of operational tools aimed at improving quality of life. In these cities, the output variables can be targeted to higher standards observed in other cities, constituting the benchmarks.
Table 7

List of cities below the 10th percentile in the efficiency assessment.

\begin{tabular}{ll}
\hline City & Efficiency score (\%) \\
\hline Verona (Italy) & 25 \\
Limoges (France) & 25 \\
Dijon (France) & 25 \\
Catania (Italy) & 25 \\
Le Havre (France) & 25 \\
Trieste (Italy) & 24 \\
Nice (France) & 24 \\
Reims (France) & 24 \\
Bari (Italy) & 24 \\
Marseille (France) & 24 \\
Palermo (Italy) & 24 \\
Napoli (Italy) & 24 \\
Taranto (Italy) & 23 \\
Ajaccio (Italy) & 23 \\
Reggio di Calábria (Italy) & 23 \\
Portsmouth (UK) & 21 \\
Moers (Germany) & 19 \\
Múrcia (Spain) & 18 \\
Frankfurt (Oder) (Germany) & 17 \\
Wrexham (UK) & 16 \\
Santander (Spain) & 10 \\
\hline
\end{tabular}

To understand the opportunities presented by this methodology of evaluation of cities efficiency, we can use Milan (Italy, capital of the North) as an illustrative example and point out where to look to improve the city performance. Table 8 presents the original values of the input and output indicators of Milan, which has an efficiency score of $25 \%$. The values of the output indicators for the peers, i.e., the efficient cities to which Milan is compared in the estimation of efficiency, are also shown. The outputs for which Milan has missing data were omitted from Table 8 . The values of the output indicators signaled by an asterisk indicate that the city has originally missing data on that indicator, which was replaced by the minimum value observed in the sample. The value of $\lambda$ shown in Table 8 for each of the peers, obtained from the optimal solution to the DEA model (6), provides an indicator of the degree of similarity between the city under assessment (Milan), and the corresponding peer.

The analysis of the data reported in Table 8 shows where the best practices for each of the outputs considered can be found. In 
Table 8

Peer cities for Milan.

\begin{tabular}{|c|c|c|c|c|c|c|}
\hline & & \multirow{2}{*}{$\begin{array}{l}\text { Observed in } \\
\text { Milan }\end{array}$} & \multicolumn{4}{|l|}{ Peers } \\
\hline & & & $\begin{array}{l}\text { Frankfurt am } \\
\text { Main }(\lambda=0.128)\end{array}$ & Erfurt $(\lambda=0.017)$ & $\begin{array}{l}\text { Helsinki } \\
(\lambda=0.818)\end{array}$ & $\begin{array}{l}\text { Tartu } \\
(\lambda=0.037)\end{array}$ \\
\hline PIB per capita & I1 & 27,119 & 27,756 & 27,756 & 27,619 & 13,539 \\
\hline Demography & 01 & 2 & 12 & 7 & $0^{*}$ & $0^{*}$ \\
\hline Social aspects & 06 & 55 & 16 & 30 & 44 & 67 \\
\hline \multirow[t]{3}{*}{ Economic aspects } & 011 & 94 & 95 & 85 & 91 & 95 \\
\hline & 012 & 94 & 96 & 85 & 93 & $61^{*}$ \\
\hline & 013 & 45 & 51 & 54 & 54 & 42 \\
\hline Education & 017 & 56 & 45 & 60 & 80 & 51 \\
\hline \multirow[t]{3}{*}{ Environment } & $\mathbf{0 2 0}$ & 323 & 339 & $297^{*}$ & $297^{*}$ & $297^{*}$ \\
\hline & 022 & 4 & 3 & 100 & $0^{*}$ & 44 \\
\hline & 023 & 11 & 23 & 191 & 16 & $0^{*}$ \\
\hline
\end{tabular}

* Missing value replaced by the minimum observed in the sample.

this illustrative case, considering the environment dimension and looking specifically to green space $(\mathrm{O} 23)$ and treatment of solid waste (022), Milan can improve its performance applying the policy of Erfurt (Germany). And in what concerns air pollution (020), the capital of North Italy has lessons to learn from Frankfurt am Main (Germany). In the training and education dimension, Italian policy-makers have to study Helsinki (Finland) strategy to include a bigger number of children between ages 0 and 4 in day care institutions (017). In what concerns the activity rate (013), Erfurt and Helsinki show an inspiring performance for Milan. This city can also use Frankfurt am Main as an example for improving its female employment rate (012) and employment rate (011). Tartu (Estonia) is an example to follow in what concerns percentage of households living in owned dwellings (06). Finally, in the demography dimension, Frankfurt and Erfurt (to a lower extension) are examples for Milan in what concerns population change over a period of 2 years ( 01$)$.

\section{Conclusion}

Urban quality of life is an issue of undeniable importance for local policy-makers. Improvements in this field can lead to a growing competitiveness of cities, but making the right decisions is not an easy task. Thus, benchmarking is an opportunity for cities to learn with other experiences, establishing performance targets that can be reproducible in order to improve quality of life for citizens.

Diverse pictures of cities efficiency, in its different aspects (such as strengths and weaknesses, contextualised performance and possible strategies for benchmarking), were presented in this paper, contributing to a discussion on a leading concern in European policies. The importance of urban quality of life for the European Union is shown by the inclusion of the Urban Audit project in the core of the Eurostat. Although the database underlying this project was not designed to be an operational tool in what concerns improvements of quality of life, it can provide fundamental information for local policy-making, namely when this data is analyzed through DEA.

As said before, cities are increasingly competing for investments and qualified human resources. But with this study of cities performance we show how cities, competing for investment and human capital (that may be more attracted by efficient cities), can cooperate in order to attain higher levels of development. Thus, with a DEA approach to urban efficiency, we can provide a useful framework to the concept of "co-opetion" (a combination of the words competition and co-operation), introduced as a major challenge for European cities by Gérard Collomb, former president of the network Eurocities, in an interview to the publication Futuribles [11].

This work shows that DEA can be used to assess quality of life of cities and to support decision making by local authorities. With this method it is possible to identify the most efficient cities in terms of quality of life and add a complementary measure of efficiency, given by the number of times they appear as an example for others. This approach also allows developing a systematic approach to the establishment of benchmarks for the less efficient cities. This provides policy-makers the opportunity to identify which urban policies can be used as an example for the dimensions that need improvements. Some examples of the potentiality of this new method were presented in this paper.

As DEA is a methodology that enables each city to obtain the best possible score by a weight assignment that emphasizes the best dimensions, no negative bias in the assessment of efficiency can be pointed out with the application of DEA. On the other hand, this approach makes clear that when a city is classified as inefficient, it means that its peers presented better performance, and that the result does not depend on subjective weight assumptions which could be criticized.

This perspective of work, with no a priori specification of weights for the dimensions considered, allows each city to present and emphasize its strengths, given its legitimate urban specialization perspective. In order to avoid extreme scenarios that could result from the non-negativity weight restrictions underlying a standard DEA formulation, the model was programmed to consider minimum assumptions in what concerns the relative importance of the outputs (a virtual weight of at least $1.5 \%$ for all dimensions). We still maintained some of the flexibility allowed by DEA, by not restricting weights inside each dimension, which still enables cities to clearly identify their strengths. This approach, besides contributing to the actual debate on composite indicators and weights restrictions, has allowed improving the discrimination of the DEA model and thus gives a more precise picture of cities performance.

Following a recent line of research in this field, we have also shown that the establishment of a ranking of cities is possible, through the construction of a composite indicator that only looks at the output achievements assessed on the basis of a common set of weights. This approach, using goal programming, makes the comparison of cities possible as it is based on equal parameters regarding the relative importance of the dimensions of quality of life.

This work also proposed to evaluate cities performance in order to promote improvements in local management, a perspective 
requiring that the resources of the city, proxied by the level of GDP per capita, are taken into consideration. Weight restrictions were further tightened, by giving equal weight to all dimensions, thus reflecting the lack of consensus in literature concerning the relative importance of the dimensions that can be considered to assess quality of life. This approach enables an assessment of cities performance in a balanced multidimensional frame, given the national wealth, and assigns peers for the cities considered inefficient.

The results of cities performance assessment here presented would obviously be different if other assumptions had been adopted. But the main purpose of this paper is achieved by the demonstration of the potential of the DEA methodology in the analysis of urban performance. What is also demonstrated is that the models here presented can be easily adjusted, in terms of the weighting system, in accordance with other perspectives of urban quality of life from different stakeholders, thus opening a door to a vast field of work.

Some limitations of this study can be pointed out. Possibly due to the fact that it is still a recent project, Urban Audit is still faced with a significant number of cases with missing data. The more this project can overcome the absence of information, the more operative will become this new tool we explored for assessing urban quality of life.

\section{References}

[1] Allen R, Athanassopoulos A, Dyson RG, Thanassoulis E. Weights restrictions and value judgments in data envelopment analysis: evolution, development and future directions. Annals of Operations Research 1997;73:13-64.

[2] Avkiran NC, Morita H. Benchmarking firm performance from a multiplestakeholder perspective with an application to Chinese banking. Omega 2010;38:501-8

[3] Banker RD, Charnes A, Cooper WW. Some models for estimating technical and scale inefficiencies in data envelopment analysis. Management Science 1994;30(9):1078-92.

[4] Beaupuy, JM. Report on the urban dimension in the context of enlargement. Committee on Regional Development, Session document of the European Parliament, 2004/2258(INI), 2005

[5] Bigcities. Quality of life in New Zealand's eight largest cities, <http://www. bigcities.govt.nz/pdfs/Quality_of_Life_2003.pdf $>; 2003$ [last visited 10/June/ 2009].

[6] Bougnol ML, Duká JH, Estellita Lins MP, Moreira da Silva AC. Enhancing standard performance practices with DEA. Omega 2010;38:33-45.

[7] Casu B, Girardone C. Integration and efficiency convergence in EU banking markets. Omega 2010;38:260-7.

[8] Charnes A, Cooper W, Rhodes E. Measuring the efficiency of decision making units. European Journal of Operational Research 1978:2:429-44.

[9] Cherchye L, Moesen W, Rogge N, Puyenbroeck T. An introduction to 'benefit of the doubt' composite indicators. Social Indicators Research 2007;82:111-45.

[10] Cherchye L, Moesen W, Rogge N, Van Puyenbroeck T, Saisana M, Saltelli A, et al. Creating composite indicators with DEA and robustness analysis: the case of the technology achievement index. Journal of Operational Research Society 2008;59:239-51.
[11] Collomb G, Haentjens J. Les villes européennes en «coopétition». Futuribles 2009;354:19-32.

[12] Despotis DK. Measuring human development via data envelopment analysis: the case of Asia and the Pacific. Omega 2005;33:385-90.

[13] European Comission. Results of the Urban Audit think tank meetings, Luxembourg, Document E4/REG/2005/02, 2005.

[14] Florida R. The rise of the creative class. New York: Basic Books; 2002.

[15] Gorman M, Ruggiero J. Evaluating state police performance using data envelopment analysis. International Journal of Production Economics 2008;113:1031-7.

[16] Hollingsworth B, Smith P. Use of ratios in data envelopment analysis. Applied Economics Letters 2003:10:733-5.

[17] Homburg C. Using data envelopment analysis to benchmark activities. International Journal of Production Economics 2001;73:51-8.

[18] IISD - International Institute for Sustainable Development. City of Winnipeg quality of life indicators, $\langle$ http://www.iisd.org/pdf/wpg.qoli.pdf $\rangle ; 1997$ [last visited 10/June/2009].

[19] Kamp IV, Leidelmeijer K, Marsman G, Hollander A. Urban environmental quality and human well-being: towards a conceptual framework and demarcation of concepts-a literature study. Landscape and Urban Planning 2003;65:5-18.

[20] Kuosmanen T. Modelling blank data entries in data envelopment analysis. Econ-WPA working paper at WUSTL, no. 0210001 (Econometrics) 2002.

[21] Lambiri D, Biagi B, Royuela V. Quality of life in the economic and urban economic literature. Social Indicators Research 2007;84:1-25.

[22] Lozano-Vivas A, Pastor JT. Do performance and environmental conditions act as barriers for cross-border banking in Europe?Omega 2010;38:275-82.

[23] Marans RW. Understanding environmental through quality of life studies: the 2001 DAS and its use of subjective and objective indicators. Landscape and Urban Planning 2003; 65: 73-83.

[24] Mitchell G, Namdeo A, Kay D. A new disease-burden method for estimating the impact of outdoor air quality on human health. Science of the Total Environment 2001;246:153-64.

[25] Pacione M. Introduction on urban environmental quality and human wellbeing. Landscape and Urban Planning 2003;65:1-3.

[26] Picavet E. Opportunities and pitfalls for ethical analysis in operations research and the management sciences. Omega 2010;37(6):1121-31.

[27] Portela MCAS, Camanho AS. Analysis of complementary methodologies for the estimation of school value added. Journal of the Operational Research Society 2010;61:1122-32.

[28] Salvaris M, Burke T, Pidgeon J, Kelman S. Social benchmarks and indicators for Victoria. Melbourne: Institute for Social Research-Swinburne University of Technology; 2000.

[29] Smirlis YG, Maragos EK, Despotis DM. Data envelopment analysis with missing values: an interval DEA approach. Applied Mathematics and Computation 2006;177(1):1-10.

[30] Trip JJ. Assessing quality of place: a comparative analysis of Amsterdam and Rotterdam. Journal of Urban Affairs 2007;29(5):501-17.

[31] Ulengin B, Ulengin F, Güvenç U. A multidimensional approach to urban quality of life: the case of Istanbul. European Journal of Operational Research $2001 ; 130: 361-74$

[32] United Nations. Human development report 2005-International cooperation at a crossroads: aid, trade and security in an unequal world, $\langle$ http://hdr. undp.org/en/media/HDR05_complete.pdf $\rangle$; 2005 [last visited 08/February/ 2010].

[33] Wong YHB, Beasley JE. Restricting weight flexibility in data envelopment analysis. Journal of the Operational Research Society 1990;41(9):829-35.

[34] Wu J, Liang L, Chen Y. DEA game cross-efficiency approach to Olympic rankings. Omega 2009;37:909-18.

[35] Zhu J. Data envelopment analysis vs. principal component analysis: an illustrative study of economic performance of Chinese cities. European Journal of Operational Research 1998;111:50-61. 